\title{
QCD thermodynamics with colour-sextet quarks
}

\author{
D. K. Sinclair*† \\ HEP Division and JTI, Argonne National Laboratory, 9700 South Cass Ave., Argonne, IL, \\ 60439, USA \\ E-mail: dks@hep.anl.gov
}

\section{J. B. Kogut *}

Department of Energy, Division of High Energy Physics, Washington, DC 20585, USA

and

Dept. of Physics - TQHN, Univ. of Maryland, 82 Regents Dr., College Park, MD 20742, USA

E-mail: jbkogut@umd.edu

We study QCD with two flavours of colour-sextet quarks as a candidate walking-Technicolor theory. We simulate lattice QCD with two flavours of colour-sextet staggered quarks at finite temperatures to observe the scales of confinement and chiral-symmetry breaking. These should give us some indication as to whether the massless theory has an infrared fixed point making it a conformal field theory, or whether it exhibits confinement and chiral symmetry breaking with a slowly varying coupling constant, i.e. 'walks'. We find that unlike the case with fundamental quarks, the deconfinement and chiral-symmetry restoration transitions are far apart. The values of $\beta=6 / g^{2}$ for both transitions increase when $T a$ is decreased from $\frac{1}{4}$ to $\frac{1}{6}$ as would be expected for finite temperature transitions of an asymptotically-free field theory. So far we see no suggestion of conformal behaviour.

The XXVII International Symposium on Lattice Field Theory

July 26-31, 2009

Peking University, Beijing, China

\footnotetext{
* Speaker.

${ }^{\dagger}$ This research was supported in part by US Department of Energy contract DE-AC02-06CH11357, and in part under a Joint Theory Institute (JTI) grant.

ॠSupported in part by a National Science Foundation grant NSF PHY03-04252.
} 


\section{Introduction}

We are interested in extensions of the standard model which have a strongly-interacting Higgs sector. The most popular of such theories are known as Technicolor theories [1, 2]. These are QCD-like theories in which the techni-pions give mass to the $W$ and $Z$ through the Higgs mechanism. Such theories need to be extended in order to also give masses to the quarks and leptons. Phenomenological difficulties with such theories can be avoided if the running coupling constant 'walks', that is evolves very slowly. Such theories are called Walking Technicolor models $[3,4,5,6]$.

The evolution of the coupling constant $g$ in QCD-like theories is described by $\beta(g)$ defined by

$$
\beta(g)=\mu \frac{\partial g}{\partial \mu}=-\beta_{0} \frac{g^{3}}{(4 \pi)^{2}}-\beta_{1} \frac{g^{5}}{(4 \pi)^{4}} \ldots
$$

where $\mu$ is the momentum scale at which the running coupling constant $g(\mu)$ is defined. $\beta_{0}, \beta_{1} \ldots$ are given by perturbation theory. If the number of flavours $N_{f}$ is small enough, $\beta_{0}, \beta_{1}>0$ and the theory is asymptotically free and confining. Chiral symmetry is spontaneously broken. For $N_{f}$ sufficiently large $\beta_{0}<0$ and asymptotic freedom and confinement are lost. Between these extremes, there is a range of $N_{f}$ for which $\beta_{0}>0, \beta_{1}<0$. If these 2 terms describe the physics, there is a second fixed point which is infrared attractive. The massless theory is then conformally invariant. There is, however, an alternative scenario; the coupling constant could become large enough that a chiral condensate forms before the would-be infrared (IR) fixed point is reached. This reduces the screening of the colour 'charge', and the coupling increases again, giving confinement. Near the would-be IR fixed point the coupling walks. Perturbation theory cannot determine whether the theory is conformal or walking. Lattice gauge theory simulations provide the only reliable method for studying these non-perturbative questions.

The advent of the LHC and increased computing power have revived interest in using lattice gauge theory simulations to study candidate conformal and walking gauge theories. A number of studies have been made of QCD with $N_{f}$ flavours of fundamental quarks, with $N_{f}$ large (as large as 17) $[7,8,9,10,11,12,13,14,15,16,17,18,19,20]$. There have also been studies of QCD with $N_{f}=2$ color-sextet (symmetric tensor) quarks [21, 22, 23], and of 2-colour QCD with $N_{f}=2$ adjoint fermions $[24,25,26,27,28]$.

We are studying a particular candidate theory, QCD with 2 colour-sextet quarks. On the lattice, we are using unimproved staggered quarks and a simple Wilson plaquette gauge action for our simulations. Exact RHMC simulations are used to tune to 2 quark flavours.

For QCD with sextet quarks, asymptotic freedom is lost at $N_{f}=3 \frac{3}{10} . \beta_{1}$ changes sign at $N_{f}=1 \frac{28}{125}$. A rainbow graph approximation predicts that a condensate forms for $N_{f}<2 \frac{163}{325}$. (See [29] for a summary of such perturbative results for $S U(N)$ For recent estimates of this boundary using other techniques, see for example [30,31]). However, preliminary lattice results of DeGrand, Shamir and Svetitsky using Wilson quarks suggest $N_{f}=2$ is conformal [21].

We are simulating thermodynamics of this theory to better understand how confinement and chiral symmetry are realized. Low statistics lattice studies of the same theory, using improved Wilson quarks have been performed by DeGrand, Shamir and Svetitsky [22]. Whereas they find coincident deconfinement and chiral-symmetry restoration transitions, we find that the chiral-symmetry 
restoration transition occurs at a much higher temperature than the deconfinement transition. Both transitions appear to be finite temperature transitions and not bulk transitions. After this we intend to simulate at zero temperature to further clarify whether it is a conformal field theory, or if it walks.

\section{Simulations and results}

We are simulating the thermodynamics of QCD with 2 sextet-quark flavours on $8^{3} \times 4,12^{3} \times 4$ and $12^{3} \times 6$ lattices. On each lattice we run at quark masses $m=0.02, m=0.01$ and $m=0.005$ in an attempt to access the chiral limit. We run at a number of couplings in the range $5.0 \leq \beta=6 / g^{2} \leq$ 7.0 to cover both the expected transitions (as well as one unexpected transition). For each $(\beta, m)$ we run for a minimum of 10,000 length-1 trajectories, increasing this to 50,000-100,000 trajectories close to the deconfinement and additional non-chiral transitions. In addition we used different starts in the deconfined region to study the remnants of the $Z_{3}$ centre symmetry. The deconfinement transition is identified by an abrupt increase in the colour-triplet Wilson Line (Polyakov Loop). The chiral phase transition occurs where the chiral condensate $(\langle\bar{\psi} \psi\rangle)$ vanishes in the massless limit.

For our $N_{t}=4$ runs, we find consistency between our $8^{3} \times 4$ and $12^{3} \times 4$ simulations for the masses we have considered, indicating that finite size effects are small. We therefore present only our $12^{3} \times 4$ measurements.

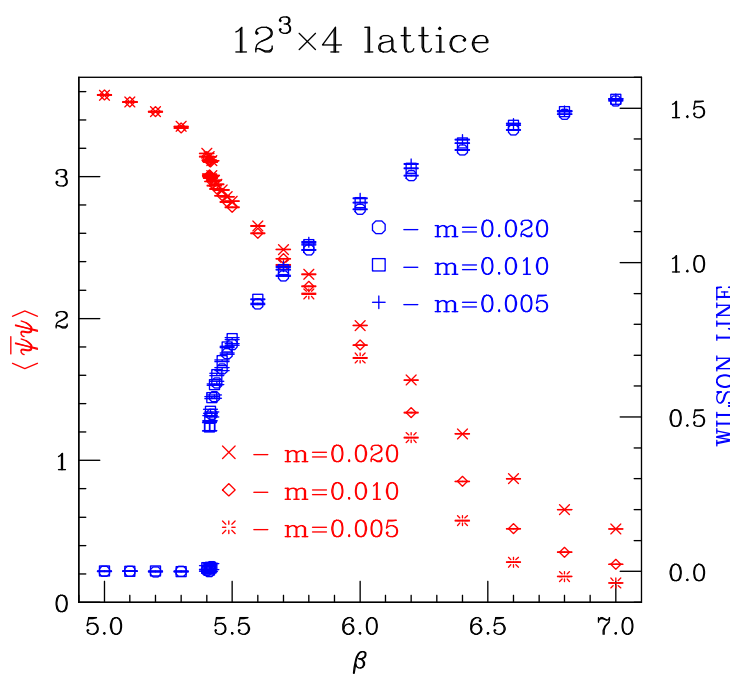

Figure 1: Wilson Lines and chiral condensates on a $12^{3} \times 4$ lattice as functions of $\beta=6 / \mathrm{g}^{2}$.

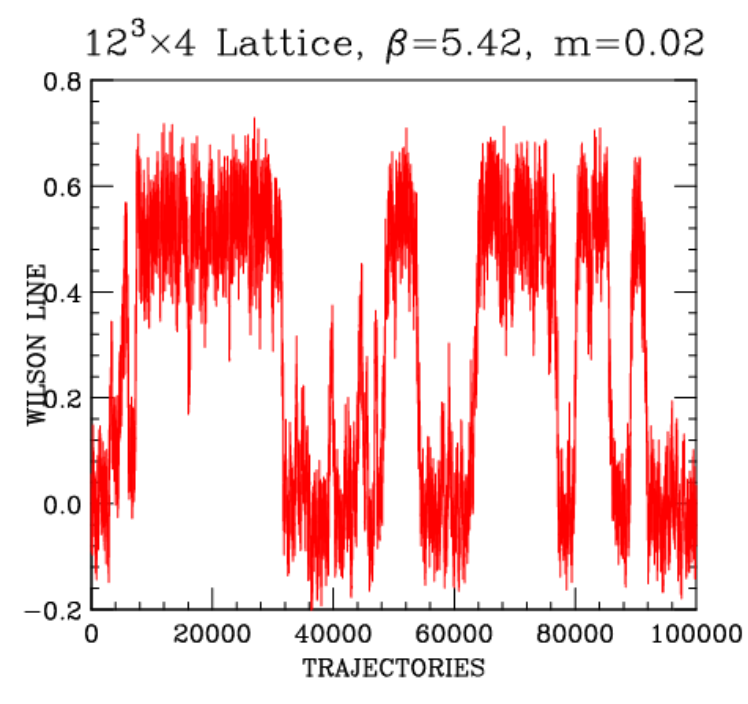

Figure 2: Evolution of the triplet Wilson line as a function of trajectory number on a $12^{3} \times 4$ lattice at $\beta=5.42, m=0.02$.

Figure 1 shows the colour-triplet Wilson Line(Polyakov Loop) and the chiral condensate $(\langle\bar{\psi} \psi\rangle)$ as functions of $\beta=6 / \mathrm{g}^{2}$, for each of the 3 quark masses on a $12^{3} \times 4$ lattice. There is clearly an abrupt transition in the Wilson Line for $\beta$ just above 5.4. Figure 2 shows the 'time' evolution of the triplet Wilson Line at $\beta=5.42, m=0.02$. The apparent 2-state signal, which is born out by histogramming, allows us to conclude that deconfinement occurs at $\beta=5.420(5)$ for $m=0.02$ and $N_{t}=4$. Similarly we find that the deconfinement transition for $m=0.01$ is at $\beta=5.412(1)$. Because the chiral-symmetry restoration phase transition is only expected for massless quarks, it is less easy to pinpoint. Our estimate for the position of this transition is at $\beta \approx 6.5$. 
Above the deconfinement transition, in addition to the state where the Wilson Line is positive, there are additional states in which the Wilson line is oriented in the direction of one of the nontrivial cube roots of unity, the remnant of the $Z_{3}$ centre symmetry. However, these states are only metastable, eventually decaying into the state with a real positive Wilson Line. The lifetimes of these states increase as $\beta$ is increased away from the transition, so that on a $12^{3} \times 4$ lattice, we have yet to observe such a decay beyond $\beta=5.46$. Above $\beta \approx 5.9$ these states with complex Wilson Lines transition to states with negative Wilson Lines.

Now let us turn our attention to $N_{t}=6$. On the $12^{3} \times 6$ lattices, the $Z_{3}$ centre symmetry is again manifest, above the deconfinement transition, and the Wilson Line shows a clear 3-state signal. Figure 3 demonstrates this at $\beta=5.58, m=0.02$, which is above this transition. This graph is a scatterplot of the Wilson Lines for 100,000 trajectories. Tunnelings occur between these 3 states in all 6 directions, implying that all 3 states are stable, in contrast to what we saw for $N_{t}=4$.

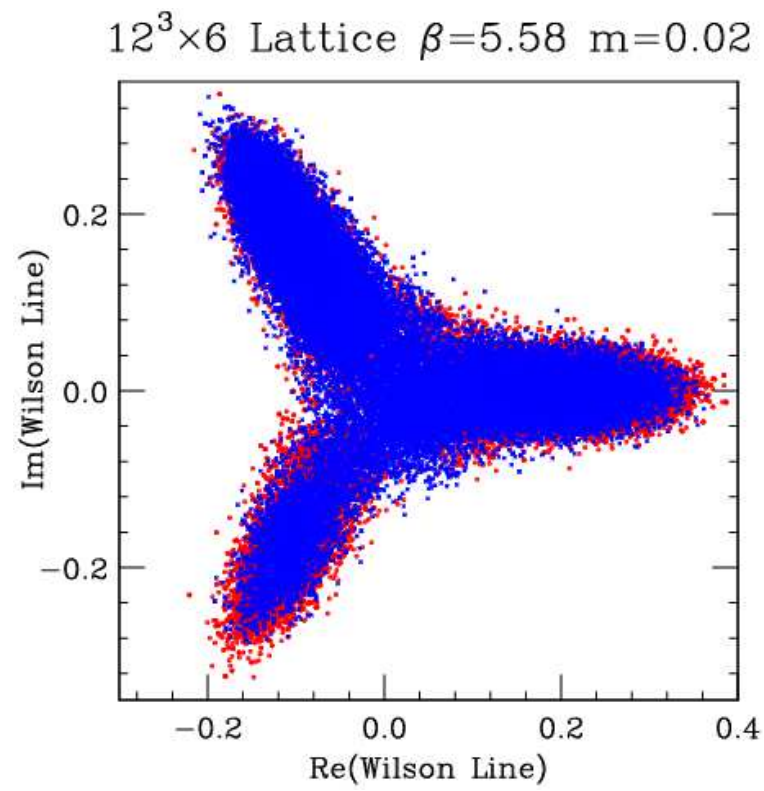

Figure 3: Scatterplot of triplet Wilson Lines at $\beta=5.58, m=0.02$, in the deconfined regime on a $12^{3} \times 6$ lattice.

We bin our observables according to whether the arguments of the Wilson Lines lie in the range $(-\pi,-\pi / 3),(-\pi / 3, \pi / 3),(\pi / 3, \pi)$. The 'data' in the first and last bins (corresponding to complex Wilson Lines) are combined, complex conjugating where necessary. Figures 4,5 show the Wilson Lines (Polyakov Loops) and the chiral condensates. The first graph is for the states with real positive Wilson Lines. The second is for those with complex (or negative) Wilson Lines. From these graphs we conclude that the deconfinement transition for $m=0.02$ occurs at $\beta=$ 5.54(4) and the chiral symmetry restoration transition is at $\beta \approx 6.8$. The increase in the $\beta$ s for each of these transitions is what would be expected if they are finite temperature transitions for an asymptotically free theory. We note that the fact that $Z_{3}$ symmetry is broken by the quarks manifests itself in the difference in magnitudes of the Wilson Line for the state with a positive and those with complex/negative Wilson Lines. 

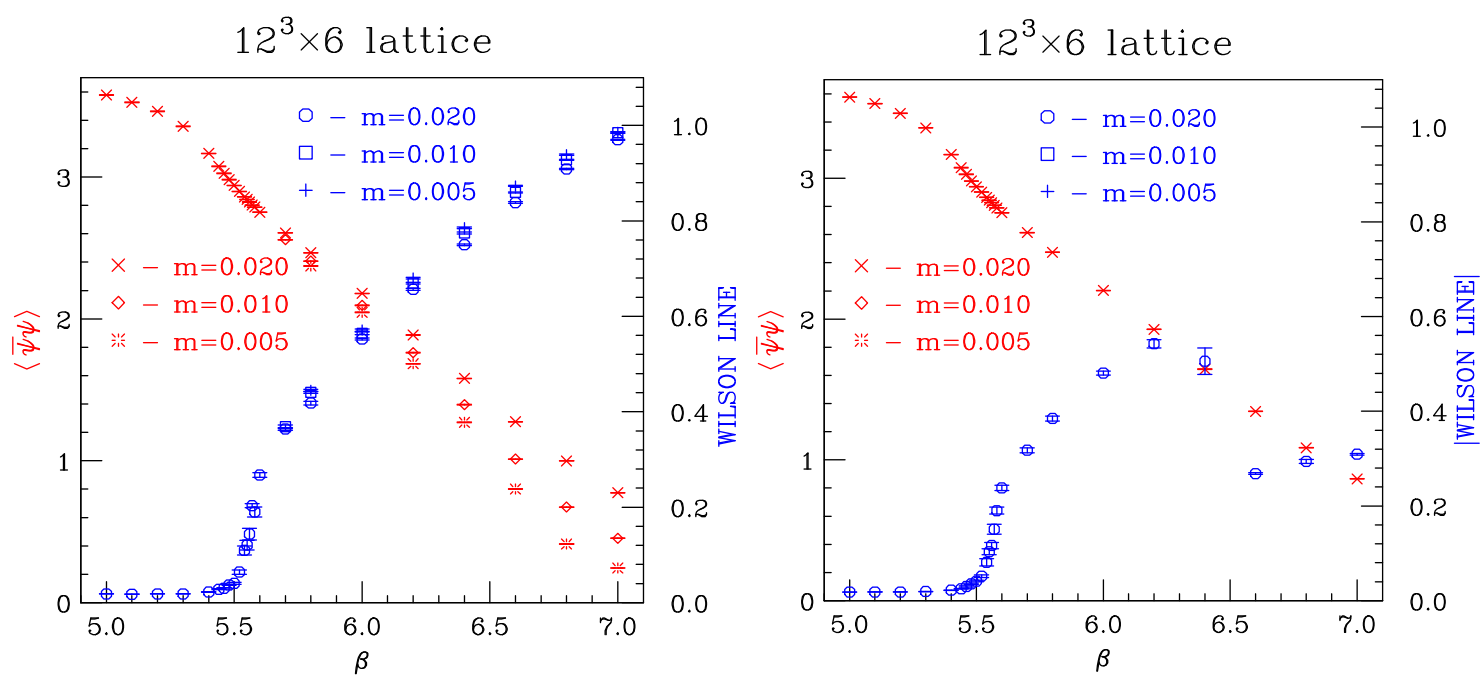

Figure 4: Triplet Wilson Line and $\langle\bar{\psi} \psi\rangle$ as functions Figure 5: Triplet Wilson Line and $\langle\bar{\psi} \psi\rangle$ as functions of $\beta$ for the state with a real positive Wilson Line. of $\beta$ for states with complex or negative Wilson Line.

The states with complex Wilson lines show a transition to states with real negative Wilson lines at $\beta \approx 6.5$. This increase over the value at $N_{t}=4$ is much larger than for the deconfinement and chiral-symmetry restoration transitions. This apparent inconsistency leads us to suspect that this particular transition is a lattice artifact.

\section{Discussion and Conclusions}

In our studies of the thermodynamics of lattice QCD with 2-flavours of colour-sextet quarks we find well separated deconfinement and chiral-symmetry restoration transitions. This contrasts with the situation with fundamental quarks where these two transitions appear coincident, but is similar to the case of adjoint quarks where again these two transitions are separate. [32, 33]

The increase in the $\beta$ s for both transitions from $N_{t}=4$ to $N_{t}=6$ is consistent with them being finite temperature transitions for an asymptotically free theory rather than bulk transitions. If this theory has an infrared stable fixed point, we have yet to observe it. Since we expect that $T_{\chi} \geq T_{d}$, the fact that $\beta_{\chi}>\beta_{d}$ is also what would be expected for an asymptotically free field theory. These very preliminary results would suggest a walking rather than a conformal behaviour.

The phase structure we observe is very different from that observed with Wilson quarks by DeGrand, Shamir and Svetitsky, who found coincident deconfinement and chiral symmetry restoration transitions. It would be possible for two different actions to give such different results if there is an infrared fixed point (as these authors find) and we are on the strong-coupling side of it. Alternatively, our quark masses might be too large to let us access the chiral limit. Because of these differences it will be interesting when simulations with sextet overlap quarks [23] are extended to larger lattices.

Let us summarise our preliminary results. At $N_{t}=4, \beta_{d}(m=0.02)=5.420(5), \beta_{d}(m=$ $0.01)=5.412(1)$; deconfinement appears to be first order. $\beta_{\chi} \approx 6.5$. At $N_{t}=6, \beta_{d}(m=0.02)=$ 5.54(4). $\beta_{\chi} \approx 6.8$. 
For the deconfined phase there is a 3-state signal, the remnant of now-broken $Z_{3}$ symmetry. For $N_{t}=4$ the states with complex Polyakov Loops appear metastable. For $N_{t}=6$ all 3 states appear stable. Breaking of $Z_{3}$ symmetry is seen in the magnitudes of the Wilson Lines for the states with positive real versus those with complex/negative Wilson lines. The existence of the 3-state signal is presumably because the formation of the chiral condensates at short distances suppresses the contribution of the quarks at the confinement scale, so the deconfined but chirally broken phase is more similar to the deconfined phase of the quenched theory. (It would be interesting to compare this to deconfined phase with heavy colour-triplet quarks).

Between the deconfinement and chiral transitions, we find a third transition where the Wilson Lines in the directions of the 2 non-trivial roots of unity change to real negative Wilson Lines. This transition occurs for $\beta \approx 5.9\left(N_{t}=4\right)$ and $\beta \approx 6.5\left(N_{t}=6\right)$. This rapid increase suggests that the transition is a lattice artifact. If this third transition is real, the fact that the magnitude of the negative Wilson line is roughly one third of that for the positive Wilson line, suggests that this transition might be associated with colour symmetry breaking $S U(3) \longrightarrow S U(2) \times U(1)$.

We need larger lattices $-18^{3} \times 6$ to study finite volume effects at $N_{t}=6$ and $16^{3} \times 8$ to see that the $N_{t}$ dependence of $\beta_{d}$ and $\beta_{\chi}$ which we observe is not a lattice artifact of these coarse lattices. Smaller quark masses are needed to access the chiral limit unambiguously. To understand this theory more fully, we need to study its zero temperature behaviour, measuring its spectrum, string tension, potential, $f_{\pi} \ldots$. Measurement of the running of the coupling constant for weak coupling is needed.

It would be useful to repeat these simulations for 3 flavours of sextet quarks, where it is believed that there should be an infrared stable fixed point, to see if we find a qualitatively different phase diagram. Lattice simulations should be applied to other candidate theories.

\section{Acknowledgements}

These simulations were performed on the Cray XT4, Franklin at NERSC under an ERCAP allocation and on the Linux cluster, Abe at NCSA under an LRAC grant.

\section{References}

[1] S. Weinberg, Phys. Rev. D 19, 1277 (1979).

[2] L. Susskind, Phys. Rev. D 20, 2619 (1979).

[3] B. Holdom, Phys. Rev. D 24, 1441 (1981).

[4] K. Yamawaki, M. Bando and K. i. Matumoto, Phys. Rev. Lett. 56, 1335 (1986).

[5] T. Akiba and T. Yanagida, Phys. Lett. B 169, 432 (1986).

[6] T. W. Appelquist, D. Karabali and L. C. R. Wijewardhana, Phys. Rev. Lett. 57, 957 (1986).

[7] J. B. Kogut, J. Polonyi, H. W. Wyld and D. K. Sinclair, Phys. Rev. Lett. 54, 1475 (1985).

[8] M. Fukugita, S. Ohta and A. Ukawa, Phys. Rev. Lett. 60, 178 (1988).

[9] S. Ohta and S. Kim, Phys. Rev. D 44, 504 (1991).

[10] S. y. Kim and S. Ohta, Phys. Rev. D 46, 3607 (1992). 
[11] F. R. Brown, H. Chen, N. H. Christ, Z. Dong, R. D. Mawhinney, W. Schaffer and A. Vaccarino, Phys. Rev. D 46, 5655 (1992) [arXiv:hep-lat/9206001].

[12] Y. Iwasaki, K. Kanaya, S. Sakai and T. Yoshie, Phys. Rev. Lett. 69, 21 (1992).

[13] Y. Iwasaki, K. Kanaya, S. Kaya, S. Sakai and T. Yoshie, Phys. Rev. D 69, 014507 (2004) [arXiv:hep-lat/0309159].

[14] A. Deuzeman, M. P. Lombardo and E. Pallante, Phys. Lett. B 670, 41 (2008) [arXiv:0804.2905 [hep-lat]].

[15] A. Deuzeman, M. P. Lombardo and E. Pallante, arXiv:0904.4662 [hep-ph].

[16] T. Appelquist, G. T. Fleming and E. T. Neil, Phys. Rev. D 79, 076010 (2009) [arXiv:0901.3766 [hep-ph]].

[17] T. Appelquist, G. T. Fleming and E. T. Neil, Phys. Rev. Lett. 100, 171607 (2008) [Erratum-ibid. 102, 149902 (2009)] [arXiv:0712.0609 [hep-ph]].

[18] X. Y. Jin and R. D. Mawhinney, PoS LATTICE2008, 059 (2008) [arXiv:0812.0413 [hep-lat]].

[19] X. Y. Jin and R. D. Mawhinney, Talk presented at Lattice 2009, Beijing (2009).

[20] Z. Fodor, K. Holland, J. Kuti, D. Nogradi and C. Schroeder, arXiv:0907.4562 [hep-lat].

[21] Y. Shamir, B. Svetitsky and T. DeGrand, Phys. Rev. D 78, 031502 (2008) [arXiv:0803.1707 [hep-lat]].

[22] T. DeGrand, Y. Shamir and B. Svetitsky, Phys. Rev. D 79, 034501 (2009) [arXiv:0812.1427 [hep-lat]].

[23] Z. Fodor, K. Holland, J. Kuti, D. Nogradi and C. Schroeder, arXiv:0809.4888 [hep-lat].

[24] S. Catterall and F. Sannino, Phys. Rev. D 76, 034504 (2007) [arXiv:0705.1664 [hep-lat]].

[25] S. Catterall, J. Giedt, F. Sannino and J. Schneible, JHEP 0811, 009 (2008) [arXiv:0807.0792 [hep-lat]].

[26] L. Del Debbio, A. Patella and C. Pica, arXiv:0805.2058 [hep-lat].

[27] L. Del Debbio, B. Lucini, A. Patella, C. Pica and A. Rago, arXiv:0907.3896 [hep-lat].

[28] A. J. Hietanen, K. Rummukainen and K. Tuominen, arXiv:0904.0864 [hep-lat].

[29] D. D. Dietrich and F. Sannino, Phys. Rev. D 75, 085018 (2007) [arXiv:hep-ph/0611341].

[30] E. Poppitz and M. Unsal, JHEP 0909, 050 (2009) [arXiv:0906.5156 [hep-th]].

[31] A. Armoni, arXiv:0907.4091 [hep-ph].

[32] F. Karsch and M. Lutgemeier, Nucl. Phys. B 550, 449 (1999) [arXiv:hep-lat/9812023].

[33] J. Engels, S. Holtmann and T. Schulze, Nucl. Phys. B 724, 357 (2005) [arXiv:hep-lat/0505008]. 